EPiC Series in Engineering
Volume 3, 2018, Pages 2342-2350
HIC 2018. 13th International
Conference on Hydroinformatics

\title{
Study of Pollutant Transport in Environmental Flows using Depth-Averaged Random Walk Method
}

\author{
Xuefei $\mathrm{Wu}{ }^{1}$, Fan Yang ${ }^{2 *}$, and Dongfang Liang ${ }^{2}$ \\ ${ }^{1}$ School of Environment and Resource, Southwest University of Science and Technology, \\ Sichuan, 621010, China \\ ${ }^{2}$ Department of Engineering, University of Cambridge, Trumpington Street, Cambridge CB2 1PZ, \\ United Kingdom \\ 13426013533 @163.com,fy250@cam.ac.uk, d. liang@eng.cam.ac.uk
}

\begin{abstract}
A depth-averaged random walk scheme is applied to investigate the process of solute transport, including advection, diffusions and reaction. Firstly, the model is used to solve an instantaneous release problem in a uniform flow, for which analytical solutions exist. Its performance is examined by comparing numerical predictions with analytical solutions. The advantage of the random walk model includes high accuracy and small numerical diffusion. Extensive parametric studies are carried out to investigate the sensitivity of the predictions to the number of particles. The result reveals that the particle number influences the accuracy of the model significantly. Finally, the model is applied to track a pollutant cloud in the Thames Estuary, where the domain geometry and bed elevation are complex. The present model is free of fictitious oscillations close to sharp concentration gradients and displays encouraging efficiency and accuracy in solving the solute transport problems in a natural aquatic environment.
\end{abstract}

\section{Introduction}

Pollutant transport in various water bodies has been studied intensively since it can give rise to a range of serious environmental problems and cause significant economic loss (Rajar, 1997). In natural environments, many water bodies can be described as shallow water, such as estuarine, coastal and inland flows. The horizontal scale of these flows is normally much larger than its depth. Therefore, it is assumed that the solute is well-mixed vertically over the water column, allowing a depth-integrated

${ }^{*}$ Corresponding author: fy250@cam.ac.uk 
approach to investigate the solute transport process. This paper focuses on the solute transportation in shallow water bodies specifically.

With the development of computing techniques, various modern numerical schemes have been applied to such problems (Lin, 1997) (Gupta I, 2004) (Begnudelli L, 2006) (Benkhaldoun F, 2007). These schemes can be classified into either Eulerian or Lagrangian methods. Previously, most of the research is based on Eulerian approaches to solve the standard advection-diffusion equation using finitedifference or finite-element techniques (Liang D F. R., 2006) (Mingham C G, 2001). However, it has been proved that these grid-based approaches are flawed in resolving steep concentration gradients, and trend to produce numerical diffusion (Liang D F. R., 2006). By contrast, Lagrangian methods use discrete massless particles as indicators to represent solute or pollutant clouds, and these particles are tracked independently in flows. Thus, the Lagrangian approach can usually yield an accurate estimation (Zhang, 2007). Nowadays, thanks to the dramatic progress in the quality and speed of computers, the computational price for the Lagrangian approach could be well paid. Its merits, such as conservative and free from artificial diffusion, become more and more outstanding. Compared with Euler methods, the Lagrangian methods are better suited to the simulation of complex phenomena, where high contamination gradients are involved.

The computational cost for Lagrangian approaches depends on two factors: the number of particles to present the pollutant cloud, and the size of the computational time step that the model applied. The choice of these two factors is crucial to the efficiency of the random walk model, and it is necessary to manually tune the number of particles and the size of the time step. This paper investigates the sensitivity of the predictions to the particle number in the depth-averaged random walk model. Firstly, the test cases consider the instantaneous release problem in uniform flows, which involves both the advection and diffusion phenomena. Then, the paper describes the applications of the random walk model to simulate a fictitious release problem over a real terrain.

\section{Depth-averaged Advection-diffusion equation}

The governing formula of solute transportation in water bodies is the advection-diffusion equation. Advection is the transportation of a substance by a fluid due to the fluid's bulk motion. In this process, we assume that the solute material exactly follows the flow. Diffusion is the process whereby mass transports from higher concentration to lower concentration in flow due to random movements. It is assumed that the diffusive substances would not affect the motion of flows. In other words, the flow field is independent of the existence of the diffusive materials. With these assumptions, the conservation of solute mass is presented as:

$$
\frac{\partial(c d)}{\partial t}+\frac{\partial(u c d)}{\partial x}+\frac{\partial(v c d)}{\partial y}=\frac{\partial}{\partial x}\left(d D_{x x} \frac{\partial c}{\partial x}+d D_{x y} \frac{\partial c}{\partial y}\right)+\frac{\partial}{\partial y}\left(d D_{y x} \frac{\partial c}{\partial x}+d D_{y y} \frac{\partial c}{\partial y}\right)+q_{s}
$$

where $t$ is time; $d$ is the water depth; $c$ is the water quality indicator, representing the depth-averaged concentration of the solute; $u$ and $v$ are the velocity components along $x$ and $y$-axis respectively; $D_{x x}$, $D_{x y}, D_{y x}$ and $D_{y y}$ constitute the dispersion-diffusion tensor of depth-averaged mixing, whose principal direction coincides with the flow direction; $q_{s}$ is the sources term of the governing equation, representing the increase $\left(q_{s}>0\right)$ or decrease $\left(q_{s}<0\right)$ in the total amount of the solute. 


\section{Depth-averaged random-walk method}

\subsection{Equation reformation}

In this paper, Eq. 1 is recast in a form that utilises a consistent particle-tracking algorithm (Józsa, 1989). A new concentration variable, $C=c d$, is introduced. Then, the depth-averaged advectiondiffusion equation can be written as:

$$
\begin{aligned}
& \frac{\partial C}{\partial t}+\frac{\partial(U C)}{\partial x}+\frac{\partial(V C)}{\partial y}=\frac{\partial^{2}\left(D_{x x} C\right)}{\partial x^{2}}+2 \frac{\partial^{2}\left(D_{x y} C\right)}{\partial x \partial y}+\frac{\partial^{2}\left(D_{y y} C\right)}{\partial y^{2}} \\
& U=u+\frac{\partial D_{x x}}{\partial x}+\frac{\partial D_{x y}}{\partial y}+\frac{D_{x x}}{d} \frac{\partial d}{\partial x}+\frac{D_{x y}}{d} \frac{\partial d}{\partial y} \\
& V=v+\frac{\partial D_{y y}}{\partial y}+\frac{\partial D_{y x}}{\partial x}+\frac{D_{y y}}{d} \frac{\partial d}{\partial y}+\frac{D_{x y}}{d} \frac{\partial d}{\partial x}
\end{aligned}
$$

The source term $q_{s}$ in Eq. 1 is neglected in Eq. 2. The concentration $C$ is considered as a probability density function. The modified advective velocities in Eq. 3 and Eq. 4 allow this particle-tracking scheme to simulate diffusion using a consistent random-walk technique. Then, the random walk model is carried out by the advective transport and the diffusive transport for each time step.

\subsection{Advective transport}

As most of the input flow field is solved by a grid-based method, it is necessary to evaluate the advective velocities in Eq. 3 and Eq. 4 at each position of particles. The advective velocities, $u$ and $v$, are computed using Taylor series expansions up to second-order about the nearest non-zero velocity node in the grid cell containing the particle. Then, a second-order accurate iterative technique is used to calculate the new particle position as:

$$
\begin{aligned}
& x_{a}(t+\Delta t)=x_{a}(t)+U \Delta t \\
& y_{a}(t+\Delta t)=y_{a}(t)+V \Delta t
\end{aligned}
$$

\subsection{Diffusive transport}

The particles are subjected to diffusion after the advective stage of a time step. The random streamwise and transverse velocity components are generated as:

$$
U_{d}^{s}=r_{1} \sqrt{\frac{2 D_{s}}{\Delta t}}, D_{s}=\varepsilon_{s} d u_{*}
$$


$U_{d}^{t}=r_{2} \sqrt{\frac{2 D_{t}}{\Delta t}}, \quad D_{t}=\varepsilon_{t} d u_{*}$

The random numbers $r_{1}$ and $r_{2}$ are independent and normally distributed with zero mean and standard deviation of unity; $D_{s}$ and $D_{t}$ are the streamwise and transverse diffusion coefficients measured parallel and perpendicular to the local velocity vector, respectively; $\varepsilon_{s}$ and $\varepsilon_{t}$ are dimensionless constants for streamwise dispersion and transverse diffusion respectively. For open channel flows in natural environments, the typical values are $\varepsilon_{s}=13.0$ and $\varepsilon_{t}=1.2$ (RA, 1991). The shear velocity $u_{*}$ can be calculated as Eq. 9 using Chézy coefficient.

$u_{*}=\frac{\sqrt{g}}{\text { Chézy }} \cdot \sqrt{u^{2}+v^{2}}$

The relationship between the streamwise-transverse system and Cartesian coordinate can be expressed as:

$$
D=\left[\begin{array}{cc}
D_{x x} & D_{x y} \\
D_{y x} & D_{y y}
\end{array}\right]=\left[\begin{array}{cc}
\cos \theta & \sin \theta \\
-\sin \theta & \cos \theta
\end{array}\right]\left[\begin{array}{cc}
D_{s} & 0 \\
0 & D_{t}
\end{array}\right]\left[\begin{array}{cc}
\cos \theta & -\sin \theta \\
\sin \theta & \cos \theta
\end{array}\right]
$$

where $\theta=\tan ^{-1}(v / u)$ is defined as the angle between the local flow direction and the $\mathrm{x}$-axis. Then, the transformation between the two coordinates can be expressed as:

$$
\begin{aligned}
& D_{x x}=D_{s} \cos ^{2} \theta+D_{t} \sin ^{2} \theta \\
& D_{x y}=D_{y x}=\left(D_{s}-D_{t}\right) \sin \theta \cos \theta \\
& D_{y y}=D_{s} \sin ^{2} \theta+D_{t} \cos ^{2} \theta \\
& U_{x}=U_{d}^{s} \cos \theta-U_{d}^{t} \sin \theta \\
& U_{y}=U_{d}^{s} \sin \theta+U_{d}^{t} \cos \theta
\end{aligned}
$$

Finally, particle's new coordinates after one-time step can be evaluated as:

$$
\begin{aligned}
& x_{\text {new }}=x_{a}(t+\Delta t)+U_{x} \Delta t \\
& y_{\text {new }}=y_{a}(t+\Delta t)+U_{y} \Delta t
\end{aligned}
$$

\subsection{Boundary condition}

Particles follow a "random walk" tratrectory with the flow inside the computational domain. The boundary treatment is designed to prevent them from crossing solid boundaries. In this study, the fully 
reflective boundary condition is applied to particles that penetrate the solid boundaries after implementing Eqs. 16-17. When the time step is not large, such a treatment then reflects the particles back into the computational domain.

\section{Model verification}

The present test case is an instantaneous release problem in uniform flows. As shown in Figure 1, the direction of the uniform flow $u=1 \mathrm{~m} / \mathrm{s}$ is along the $x$-axis $(\theta=0)$ and the water depth is set to be $H$ $=1 \mathrm{~m}$. The test domain extends from 0 to $800 \mathrm{~m}$ in both the $\mathrm{x}$ and $\mathrm{y}$ directions to eliminate boundary effects. The total amount of solute material $M=233.06 \mathrm{~kg}$ is released suddenly at the location $\left(x_{0}, y_{0}\right)$ $=(0,400 \mathrm{~m})$ when $t=0 \mathrm{~s}$. The Chézy coefficient, in this case, is fixed at $40 \mathrm{~m}^{1 / 2} / \mathrm{s}$, and the time step is 1s. The streamwise dispersion and lateral diffusion constants at 13.0 and 1.2 respectively and the mixing coefficients are calculated to be $D_{x x}=1.02 \mathrm{~m}^{2} / \mathrm{s}$ and $D_{y y}=0.094 \mathrm{~m}^{2} / \mathrm{s}$. In the following discussion, $P_{0}=$ 233 and $P$ represent the total number of particle used in the model.

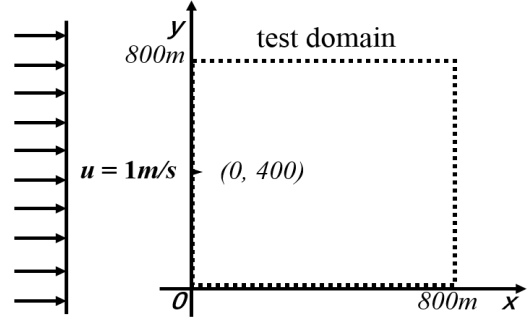

Figure 1: The instantaneous release problem in a uniform flow

Figure 2 shows a qualitative illustration of the solute transport process predicted by the random walk model with different configurations of particle number. It is notable that the major axis of the ellipses patches is in line with the flow direction, and the cloud experienced rapid elongation in the path. The reason is that the magnitude of the streamwise dispersion is more than ten times larger than that of the lateral diffusion. In general, when the particle number increases, the contour turns out to be more notable. The simulation cannot generate the significant contours when the particle number is small. Larger particle numbers significantly improve the visual inspection of the elliptical patches for solute distribution.
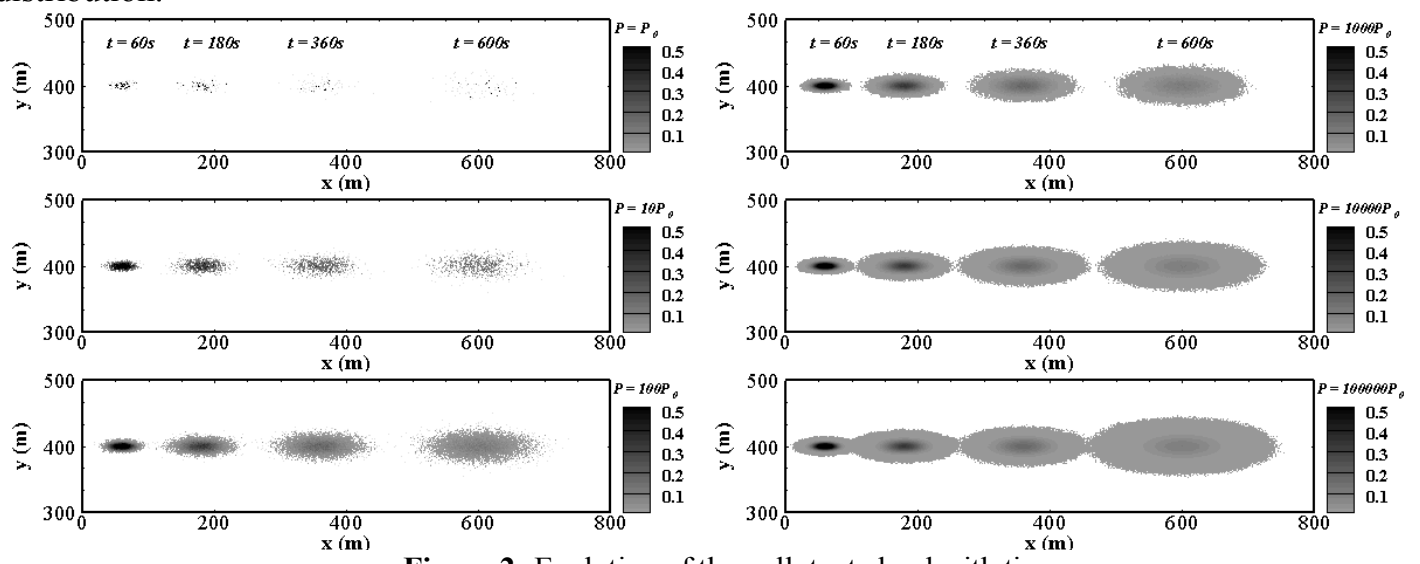

Figure 2: Evolution of the pollutant cloud with time 


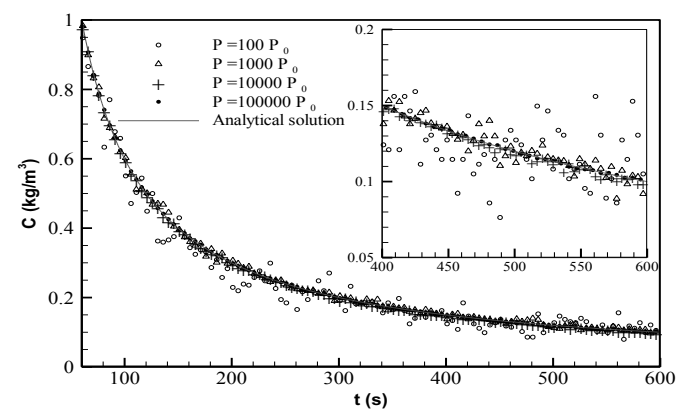

Figure 3: Variation of peak concentration with time

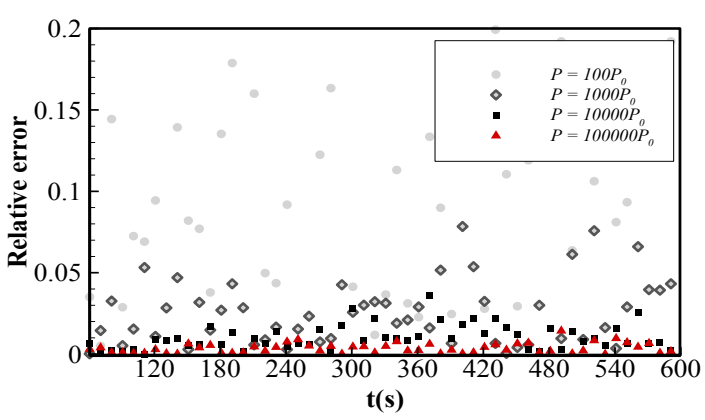

Figure 4: Variation of relative error with time

To get a quantitative analyse, the predicted results are compared with analytical solutions. For this case, the analytical solution can be expressed as:

$$
C(x, y, t)=\frac{M / H}{4 \pi t \sqrt{D_{x x} D_{y y}}} e^{-\frac{\left(x-x_{0}-u t\right)^{2}}{4 D_{x x} t}-\frac{\left(y-y_{0}\right)}{4 D_{y y} t}}
$$

As shown in Figure 3, variations of peak concentration with different particle numbers are compared with theoretical values. Small particle numbers tend to produce numerical oscillations. On the contrary, by setting a more substantial number of particles in the model, the prediction approaches closer to the analytical solution. To show this convergence trend more clearly, a subfigure of the interval between 400s and 600s is included in Figure 3. The relative errors with different particle number configurations are compared in Figure 4. The error for small particle numbers jumps beyond $20 \%$, which is unacceptable in a strict simulation. When the particle number in the model is larger than $10 \mathrm{k}$ times $P_{0}$, the error decreases below 5\%. Also, it can be seen that the relative error distribution is not affected by the concentration gradient.

\section{Application to Thames Estuary}

After being tested for the idealised case, the random walk model is applied to simulate a fictitious release problem in the Thames estuary. The geometry of this real terrain is complex, with extensive wetting and drying during tidal oscillations. At the initial stage, a passive substance is discharged into 
the water body from the point Q, as shown in Fig. 5(a). The detailed topography information about this area and physical parameters settings, such as Manning's roughness and the rate of release, can be found in (Liang D W. X., 2010).

A sequence of the concentration snapshots across the domain simulated by the random walk model are presented in Figure 5. As expected, the pollutant cloud oscillates back and forth with the tidal currents. In the first tidal circle after release, the material spreads over a broader area and becomes less concentrated as time progresses. For example, the ebbing stage can be seen in figures a, c, and e, while the flooding stage can be seen in figures $b, d$, and $f$. These rational results indicate that the current random walk model can be applied to real-world cases with an unsteady flow and complex terrain. The grid-based TVD-Mac method had been applied to the same case in Liang (Liang D W. X., 2010). By comparison, the random walk model gives an even higher resolution of the concentration distribution, as this grid-free approach is theoretically able to calculate concentration at any given location.
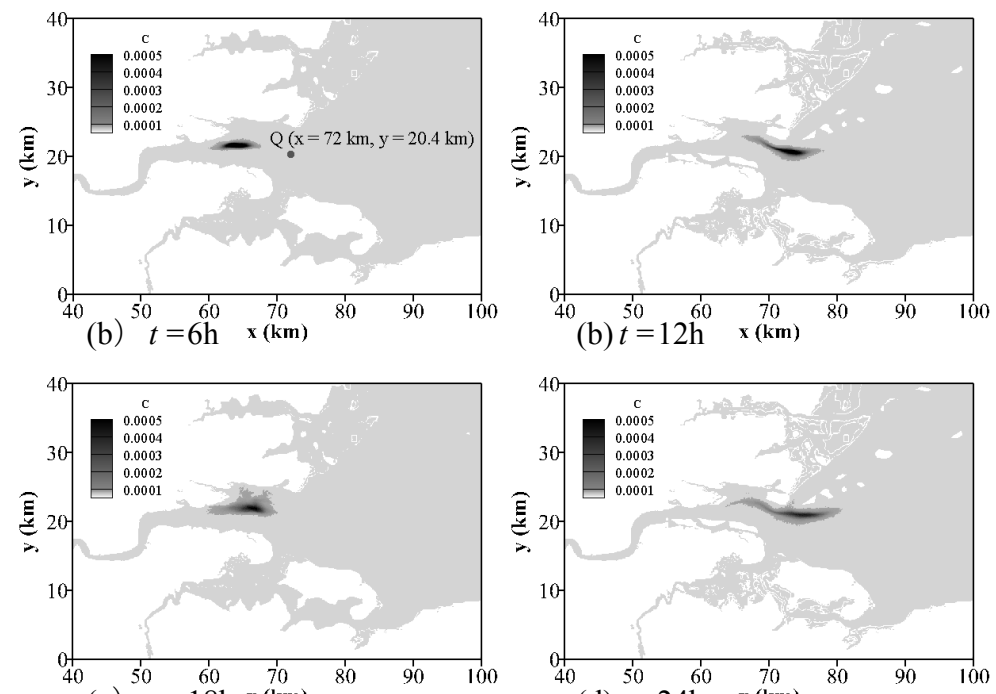

(a) $t=18 \mathrm{~h} \times(\mathbf{k m})$

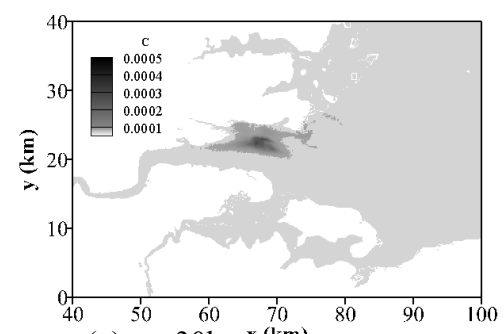

(d) $t=24 \mathrm{~h} \quad \times(\mathbf{k m})$

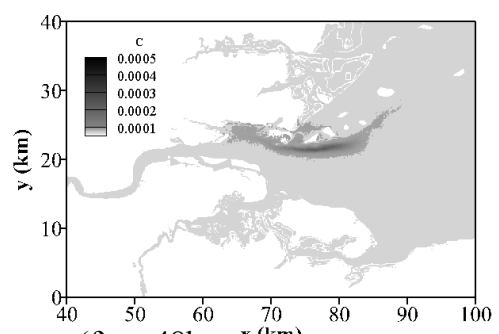

(e) $t=30 \mathrm{~h} \quad \times r k m$

(f) $t=48 \mathrm{~h}$

Figure 5 Random walk simulation of the development of pollutant concentration field for the Thames Estuary

\section{Conclusions}

The traditional random walk model has been extended to solve the depth-integrated advectiondiffusion equation. Firstly, the model is verified by solving an instantaneous release problem in a 
uniform flow. Analytical solutions are used as a reference. The results reveal several merits of this model, including high accuracy and small numerical diffusion. Extensive parametric studies have been carried out to investigate the sensitivity of the predictions to the computational parameters. It has been found that too few particles not only degrade the visual inspection of solute distribution but also produce unacceptable errors. On the contrary, simulations with sufficient particle numbers achieve good accuracy with little numerical dissipation. This computational parameter significantly influences the performance of the current random walk model. In the application, the particle number requires to be carefully chosen. Finally, the model is successfully applied to solve a hypothetical accident of pollutant release in the Thames estuary, where the domain geometry is complex and frequent flooding and drying are observed during a typical tide. The results demonstrate reasonable features of the temporal and spatial variations, which agree with the previous results based on fine meshes. The future work will be concentrated on the parametric studies concerning time steps. In practical applications, one needs to have a comprehensive understanding of the sensitivity of the predictions to computational parameters.

\section{Acknowledgements}

The study was supported by the British Council Newton Fund (UK-275929921) and the China Scholarship Council (CSC, No.201708060090).

\section{References}

Begnudelli L, S. B. (2006). Unstructured grid finite-volume algorithm for shallow-water flow and scalar transport with wetting and drying. Journal of hydraulic engineering, 132(4), pp. 371-384.

Benkhaldoun F, E. I. (2007). Well-balanced finite volume schemes for pollutant transport by shallow water equations on unstructured meshes. Journal of computational physics, 226(1), pp. 180203.

Gupta I, D. S. (2004). Numerical modeling for Thane creek. Environmental Modelling \& Software, 19(6), pp. 571-579.

Józsa. (1989). 2D particle model for predicting depth-integrated pollutant and surface oil slick transport in rivers. In Proc. of the 1st Int. Conf. on Hydraulic and Environmental Modelling of Coastal, Estuarine and River Waters, (pp. 332-340).

Liang D, F. R. (2006). Comparison between TVD-MacCormack and ADI-type solvers of the shallow water equations. Advances in water resources, 29(12), pp. 1833-1845.

Liang D, W. X. (2010). Solving the depth-integrated solute transport equation with a TVDMacCormack scheme. Environmental Modelling \& Software, 25(12), pp. 1619-1629.

Lin, B. \&. (1997). Tidal flow and transport modeling using ULTIMATE QUICKEST scheme. Journal of hydraulic engineering, 123(4), pp. 303-314.

Mingham C G, C. D. (2001). A TVD MacCormack scheme for transcritical flow. Proceedings of the Institution of Civil Engineers-Water and Maritime Engineering. 148, pp. 167-175. Thomas Telford Ltd.

RA, F. (1991). Review of modelling flow and pollutant transport processes in hydraulic basins. Springer, Dordrecht.

Rajar, R. (1997). The role of mathematical models, physical models and field measurements in water pollution problems. WIT Press.

Zhang, Z. a. (2007). Comparison of the Eulerian and Lagrangian methods for predicting particle transport in enclosed spaces. Atmospheric Environment, 41(25), pp. 5236-5248. 
$\mathrm{X}$. Wu et al. 